\title{
Reverse mathematics, trichotomy, and dichotomy
}

\author{
FRANÇOIS G DORAIS \\ JEFFRY L HIRST \\ PAUL SHAFER
}

\begin{abstract}
Using the techniques of reverse mathematics, we analyze the logical strength of statements similar to trichotomy and dichotomy for sequences of reals. Capitalizing on the connection between sequential statements and constructivity, we find computable restrictions of the statements for sequences and constructive restrictions of the original principles.
\end{abstract}

2010 Mathematics Subject Classification 03B30, 03 F35 (primary); 03F60, 30F50 (secondary)

Keywords: reverse mathematics, real numbers, sequence, WKL, ACA, Heyting arithmetic, constructive, LPO, LLPO, persistent, contractive

\section{Axiom systems and encoding reals}

We will examine several statements about real numbers and sequences of real numbers in the framework of reverse mathematics and in some formalizations of weak constructive analysis. From reverse mathematics, we will concentrate on the axiom systems $R C A_{0}$, $W K L_{0}$, and $A C A_{0}$, which are described in detail by Simpson [8]. Very roughly, $R C A_{0}$ is a subsystem of second order arithmetic incorporating ordered semi-ring axioms, a restricted form of induction, and comprehension for $\Delta_{1}^{0}$ definable sets. The axiom system $\mathrm{WKL}_{0}$ appends König's tree lemma restricted to $0-1$ trees, and the system $A C A_{0}$ appends a comprehension scheme for arithmetically definable sets. $A C A_{0}$ is strictly stronger than $W K L_{0}$, and $W K L_{0}$ is strictly stronger than $R C A_{0}$.

We will also make use of several formalizations of subsystems of constructive analysis, all variations of $\mathrm{E}-\mathrm{HA}{ }^{\omega}$, which is intuitionistic arithmetic (Heyting arithmetic) in all finite types with an extensionality scheme. Unlike the reverse mathematics systems which use classical logic, these constructive systems omit the law of the excluded middle. For example, we will use extensions of $\widehat{\mathrm{E}-\mathrm{HA}}_{\uparrow}^{\omega}$, a form of Heyting arithmetic with 
primitive recursion restricted to type 0 objects, and induction restricted to quantifier free formulas. We will often append choice axioms. For example, we will use full choice:

$$
\text { AC : } \forall x \exists y A(x, y) \rightarrow \exists Y \forall x A(x, Y(x))
$$

where $x$ and $y$ may be of any finite type. We will also use quantifier free choice, $\mathrm{QF}-\mathrm{AC} \mathrm{C}^{0,0}$, which is defined by the same scheme but where $x$ and $y$ are restricted to natural number variables and $A$ is restricted to quantifier free formulas. These systems and many others are treated in detail by Kohlenbach [7]; a short summary appears in the paper of Hirst and Mummert [5].

Both Simpson [8] (following Definition II.4.4) and Kohlenbach [7] (in section 4.1) encode real numbers as rapidly converging sequences of rational numbers. In particular, Simpson defines a real number as a Cauchy sequence of rationals $\alpha=\langle\alpha(k)\rangle_{k \in \mathbb{N}}$ satisfying $\forall k \forall i\left(|\alpha(k)-\alpha(k+i)| \leq 2^{-k}\right)$. Kohlenbach's reals are required to converge slightly more quickly. For rational numbers, equality and inequality can be expressed using quantifier free formulas of arithmetic. The situation for reals is more complicated. Two reals $\alpha$ and $\beta$ are said to be equal if $\forall k\left(|\alpha(k)-\beta(k)| \leq 2^{-k+1}\right)$. Since $\alpha(k)$ and $\beta(k)$ are rationals, this formalization of equality contains only the leading universal quantifier. Because we consider both classical and intuitionistic systems, we need to be especially careful in defining inequality for reals. For reals $\alpha$ and $\beta$, we say $\alpha<\beta$ (or $\beta>\alpha$ ) if $\exists k\left(\beta(k)-\alpha(k)>2^{-k+1}\right)$. We say that $\beta \leq \alpha$ if $\neg(\alpha<\beta)$, which is equivalent to $\forall k\left(\beta(k)-\alpha(k) \leq 2^{-k+1}\right)$ over $\widehat{\mathrm{E}-\mathrm{HA}}_{\uparrow}^{\omega}$. Note that $\beta \leq \alpha$ is not defined as a disjunction. These definitions are equivalent over $\mathrm{RCA}_{0}$ to those following Definition II.4.4 of Simpson [8]: his $\alpha<\beta$ is our $\neg \neg(\alpha<\beta)$. We say $\alpha$ is positive if $\alpha>0$ and negative if $\alpha<0$. Here 0 can be the sequence of zeros, or equivalently any real that is equal to that real. We say $\alpha$ is non-positive if $\alpha$ is not positive, which is equivalent over $\widehat{\mathrm{E}-\mathrm{HA}}_{\mid}^{\omega}$ to $\alpha \leq 0$. Similarly, $\alpha$ is non-negative means $0 \leq \alpha$. Summarizing, we have the following:

- $\alpha$ is a real means $\forall k \forall i\left(|\alpha(k)-\alpha(k+i)| \leq 2^{-k}\right)$.

- $\alpha=\beta$ means $\forall k\left(|\alpha(k)-\beta(k)| \leq 2^{-k+1}\right)$.

- $\alpha<\beta$ means $\exists k\left(\beta(k)-\alpha(k)>2^{-k+1}\right)$.

- $\beta \leq \alpha$ means $\neg(\alpha<\beta)$, or equivalently, $\forall k\left(\beta(k)-\alpha(k) \leq 2^{-k+1}\right)$.

- $\alpha$ is positive means $\alpha>0$, or equivalently, $\exists k\left(\alpha(k)>2^{-k+1}\right)$.

- $\alpha$ is negative means $\alpha<0$, or equivalently, $\exists k\left(\alpha(k)<-2^{-k+1}\right)$.

- $\alpha$ is non-positive means $\neg(\alpha>0)$, which is equivalent to $\alpha \leq 0$, which is equivalent to $\forall k\left(\alpha(k) \leq 2^{-k+1}\right)$.

- $\alpha$ is non-negative means $\neg(\alpha<0)$, which is equivalent to $\alpha \geq 0$, which is equivalent to $\forall k\left(\alpha(k) \geq-2^{-k+1}\right)$. 


\section{Reverse mathematics}

The principle of dichotomy for reals asserts that $\forall \alpha(\alpha \geq 0 \vee \alpha \leq 0)$. Since $\mathrm{RCA}_{0}$ includes the law of the excluded middle, it proves dichotomy for individual reals. However, dichotomy for a sequence of reals requires the use of weak König's lemma, as shown by the following theorem.

Theorem $1 \quad\left(\mathrm{RCA}_{0}\right)$ The following are equivalent:

(1) $W K L_{0}$.

(2) If $\left\langle\alpha_{i}\right\rangle_{i \in \mathbb{N}}$ is a sequence of reals, then there is a set $I \subset \mathbb{N}$ such that for all $i$, $i \in I$ implies $\alpha_{i} \leq 0$ and $i \notin I$ implies $\alpha_{i} \geq 0$.

Proof Working in $\mathrm{RCA}_{0}$, item 2 is easily deduced from $\Sigma_{1}^{0}$-separation, which is provable in $\mathrm{WKL}_{0}$ as shown in Lemma IV.4.4 of Simpson [8].

The same lemma of Simpson shows that $\mathrm{WKL}_{0}$ is equivalent to the separation of ranges of injections with disjoint ranges. We use this to deduce $W K L_{0}$ from item 2. Let $f: \mathbb{N} \rightarrow \mathbb{N}$ and $g: \mathbb{N} \rightarrow \mathbb{N}$ be one-to-one functions such that $\forall i \forall j(f(i) \neq g(j))$. Write $f[s]$ for the finite set $\{f(0), \ldots, f(s-1)\}$. Note that $\mathrm{RCA}_{0}$ suffices to find the finite set $f[s]$ and, since $f$ is an injection, to calculate $f^{-1}(i)$ given that $i \in f[s]$. Define the sequence $\left\langle\alpha_{i}\right\rangle_{i \in \mathbb{N}}$ by

$$
\alpha_{i}(s)= \begin{cases}2^{-f^{-1}(i)} & \text { when } i \in f[s], \\ -2^{-g^{-1}(i)} & \text { when } i \in g[s], \\ 0 & \text { otherwise. }\end{cases}
$$

It is clear that $\left\langle\alpha_{i}\right\rangle_{i \in \mathbb{N}}$ is a sequence of real numbers. Moreover, we know that $\alpha_{i}>0$ if and only if $i$ is in the range of $f$, and $\alpha_{i}<0$ if and only if $i$ is in the range of $g$. If $I \subset \mathbb{N}$ satisfies item 2, then $I$ contains the range of $g$ and excludes the range of $f$.

The main theorems of Hirst and Mummert [5] show that for certain formulas, if the formula is provable in a constructive setting then $\mathrm{RCA}_{0}$ proves a related formula for sequences. Since the preceding theorem shows that dichotomy for sequences of reals implies $\mathrm{WKL}_{0}$ (and so is not provable in $\mathrm{RCA}_{0}$ ), we may conclude that dichotomy for single reals is not provable in (an extension of) a constructive axiom system. The axiom system in the following corollary appends an extensionality scheme, full axiom of choice, and independence of premise for $\exists$-free formulas to Heyting arithmetic. It is a proper extension of a formalization of constructive analysis. 
Corollary $2 \mathrm{E}-\mathrm{HA} \mathrm{A}^{\omega}+\mathrm{AC}+\mathrm{IP}_{\mathrm{ef}}^{\omega}$ does not prove that if $\alpha$ is a real then $\alpha \geq 0$ or $\alpha \leq 0$.

Proof Apply the contrapositive of Theorem 3.6 of Hirst and Mummert [5] to a formula asserting that for every $\alpha$ there is a natural number $n$ such that if $\alpha$ is a real then either $n=0$ and $\alpha \leq 0$, or $n=1$ and $\alpha \geq 0$. This formula is in the class $\Gamma_{1}$, so the theorem applies. Theorem 1 shows that $\mathrm{RCA}_{0}$ does not prove the sequential version, so $\mathrm{E}-\mathrm{HA} \mathrm{A}^{\omega}+\mathrm{AC}+\mathrm{IP}_{\mathrm{ef}}^{\omega}$ does not prove dichotomy.

The non-constructive nature of dichotomy can also be deduced from the well-known fact that dichotomy for reals is equivalent to Bishop's lesser limited principle of omniscience. Discussions of this can be found in Bridges and Richman [2] and in Bridges and Vîţă [3]. For completeness we present a version of this fact, formulated in the fashion of a reverse mathematics result.

Theorem $3\left(\widehat{\mathrm{E}-\mathrm{HA}}_{\mid}^{\omega}+\mathrm{QF}-\mathrm{AC} \mathrm{C}^{0,0}\right)$ The following are equivalent:

(1) LLPO (Lesser limited principle of omniscience) If $f: \mathbb{N} \rightarrow\{0,1\}$ is a function that takes the value 1 at most once, then either $\forall n(f(2 n)=0)$ or $\forall n(f(2 n+1)=0)$.

(2) If $\alpha$ is a real number, then $\alpha \geq 0$ or $\alpha \leq 0$.

Consequently, neither of these statements are provable in $\mathrm{E}-\mathrm{HA}{ }^{\omega}+\mathrm{AC}$.

Proof We sketch the equivalence working in $\widehat{\mathrm{E}-\mathrm{HA}}_{\Gamma}^{\omega}+\mathrm{QF}-\mathrm{AC}^{0,0}$. Assume LLPO and suppose $\alpha$ is a real. Use QF-AC ${ }^{0,0}$ to define $f$ as follows. Set the values of $f$ to 0 as long as $|\alpha(n)| \leq 2^{-n+1}$. If we discover a (least) $k$ such that $|\alpha(k)|>2^{-k+1}$, let $f(2 k)=1$ if $\alpha(k)>0$, let $f(2 k+1)=1$ if $\alpha(k)<0$, and set all other values of $f$ to 0 . Note that if $\forall n(f(2 n)=0)$ then $\alpha \leq 0$, and if $\forall n(f(2 n+1)=0)$ then $\alpha \geq 0$.

To prove the converse, suppose $f: \mathbb{N} \rightarrow\{0,1\}$ takes the value 1 at most once. Define $\alpha$ by specifying that

- $\alpha(n)=0$ if $f(t)=0$ for all $t \leq n$,

- $\alpha(n)=2^{-t}$ if there is an even $t \leq n$ such that $f(t)=1$, and

- $\alpha(n)=-2^{-t}$ if there is an odd $t \leq n$ such that $f(t)=1$.

Note that if $\alpha \geq 0$ then $\forall n(f(2 n+1)=0)$, and if $\alpha \leq 0$ then $\forall n(f(2 n)=0)$.

The last sentence of the theorem is provable by a realizability argument by applying Corollary 2. 
Theorem 1 and Theorem 3 indirectly link weak König's lemma and LLPO. Other connections can be found in the work of Ishihara [6] and Brattka and Gherardi [1].

Following the pattern of our work with dichotomy, we now turn to trichotomy for reals: $\forall \alpha(\alpha<0 \vee \alpha=0 \vee \alpha>0)$. Since $\mathrm{RCA}_{0}$ includes the law of the excluded middle, it proves trichotomy for a single real, as noted in Section II.4 of Simpson [8]. As shown below, trichotomy for sequences of reals is equivalent to arithmetical comprehension, and consequently is strictly stronger than dichotomy for sequences.

Theorem $4 \quad\left(\mathrm{RCA}_{0}\right)$ The following are equivalent:

(1) $\mathrm{ACA}_{0}$

(2) If $\left\langle\alpha_{i}\right\rangle_{i \in \mathbb{N}}$ is a sequence of reals, then there are sets of natural numbers $L, E$, and $G$ such that $i \in L$ implies $\alpha_{i}<0, i \in E$ implies $\alpha_{i}=0$, and $i \in G$ implies $\alpha_{i}>0$.

(3) If $\left\langle\alpha_{i}\right\rangle_{i \in \mathbb{N}}$ is a sequence of non-negative reals, then there is a set $I \subset \mathbb{N}$ such that for all $i, i \in I$ implies $\alpha_{i}=0$ and $i \notin I$ implies $\alpha_{i}>0$.

Proof It is clear that arithmetic comprehension suffices to prove the existence of the sets $L, E$, and $G$ in item 2 . Since item 3 is a restriction of item 2, we can complete the proof by showing that item 3 implies $A_{C A}$.

By Lemma III.1.3 of Simpson [8], we need only show that item 3 suffices to prove the existence of ranges of injections. Let $f: \mathbb{N} \rightarrow \mathbb{N}$ be one-to-one. Using the bracket notation from the proof of Theorem 1, define $\left\langle\alpha_{i}\right\rangle_{i \in \mathbb{N}}$ by

$$
\alpha_{i}(s)= \begin{cases}2^{-f^{-1}(i)} & \text { when } i \in f[s], \\ 2^{-s} & \text { otherwise. }\end{cases}
$$

Straightforward arguments show that $\left\langle\alpha_{i}\right\rangle_{i \in \mathbb{N}}$ is a sequence of non-negative real numbers. Moreover, we see that $\alpha_{i}>0$ if and only if $i$ is in the range of $f$. Thus the range of $f$ exists by applying $\Delta_{1}^{0}$ comprehension to find the complement of the set $I$ as provided in item 3.

The next corollary follows immediately by a proof that is almost identical to that of Corollary 2.

Corollary $5 \mathrm{E}-\mathrm{HA} \mathrm{A}^{\omega}+\mathrm{AC}+\mathrm{IP}_{\mathrm{ef}}^{\omega}$ does not prove that if $\alpha$ is a real and $\alpha \geq 0$, then $\alpha>0$ or $\alpha=0$. 
Imitating Theorem 3, we present the following well known relationship between trichotomy and Bishop's limited principle of omniscience.

Theorem $6\left(\widehat{\mathrm{E}-\mathrm{HA}}^{\omega}+\mathrm{QF}-\mathrm{AC} \mathrm{C}^{0,0}\right)$ The following are equivalent:

(1) LPO (limited principle of omniscience) If $f: \mathbb{N} \rightarrow\{0,1\}$ then either $\exists n(f(n)=$ 1) or $\forall n(f(n)=0)$.

(2) If $\alpha$ is a real number and $\alpha \geq 0$, then either $\alpha>0$ or $\alpha=0$.

(3) If $\alpha$ is a real number, then $\alpha<0, \alpha=0$, or $\alpha>0$.

Consequently, none of these statements are provable in $\mathrm{E}-\mathrm{HA}{ }^{\omega}+\mathrm{AC}$.

Proof We sketch the equivalences, working in $\widehat{\mathrm{E}-\mathrm{HA}}^{\omega}+\mathrm{QF}-\mathrm{AC}^{0,0}$. First assume LPO and suppose $\alpha \geq 0$. Use QF-AC ${ }^{0,0}$ to define $f$ by $f(n)=1$ if $\alpha(n)>2^{-n+1}$ and $f(n)=0$ otherwise. Apply LPO to determine if $\alpha>0$ or $\alpha=0$.

Next, assume item 2 and let $\alpha$ be a real. Define reals $\rho$ and $\sigma$ by

$$
\rho(n)=\left\{\begin{array}{ll}
\alpha(n) & \text { if } \alpha(n) \geq 0, \\
0 & \text { otherwise. }
\end{array} \quad \text { and } \quad \sigma(n)= \begin{cases}-\alpha(n) & \text { if } \alpha(n) \leq 0, \\
0 & \text { otherwise }\end{cases}\right.
$$

Note that $\rho$ and $\sigma$ satisfy the convergence rate requirements for reals, and that both $\rho \geq 0$ and $\sigma \geq 0$. Apply item 2 to $\rho$ and $\sigma$. If $\rho>0$, then $\alpha>0$. If $\sigma>0$, then $\alpha<0$. If both $\rho=0$ and $\sigma=0$, then $\alpha=0$. Thus, item 3 holds.

Finally, assume item 3 and suppose $f: \mathbb{N} \rightarrow\{0,1\}$. Define $\alpha$ as follows. For each $n$, let $\alpha(n)=0$ if $f(t)=0$ for all $t \leq n$, and let $\alpha(n)=2^{-t}$ if $t$ is the least number less than or equal to $n$ such that $f(t)=1$. Then $\alpha \geq 0$. Applying item 3, if $\alpha=0$ then $\forall n(f(n)=0)$ and if $\alpha>0$ then $\exists n(f(n)=1)$.

The final sentence of the theorem may be derived directly from realizability arguments, or proved by applying Corollary 5 .

\section{Restrictions}

The goal of this section is to define subclasses of reals for which dichotomy and trichotomy can be proved constructively. In light of the result of Hirst and Mummert [5], the corresponding sequential restrictions will be provable in $\mathrm{RCA}_{0}$. We begin by addressing dichotomy. 
Definition 7 Let $\alpha$ be a real number.

- We say that $\alpha$ is upper $k$-persistent if

$$
\forall s(s \geq k \wedge \alpha(s) \geq 0 \rightarrow \exists t(t>s \wedge \alpha(t) \geq 0)) .
$$

- We say that $\alpha$ is lower $k$-persistent if

$$
\forall s(s \geq k \wedge \alpha(s) \leq 0 \rightarrow \exists t(t>s \wedge \alpha(t) \leq 0)) .
$$

- We say that $\alpha$ is $k$-persistent if it is both upper and lower $k$-persistent.

Informally, if a rational sequence is $k$-persistent, after the first $k$ entries, the tail contains no last non-positive entry and no last non-negative entry. Every positive real has a tail that consists entirely of positive rationals, so if the $k^{\text {th }}$ entry of a $k$-persistent real is nonpositive, then the real cannot be positive. Since the real is not positive, it is non-positive. Similarly, if the $k^{\text {th }}$ entry is non-negative, then the real must be non-negative. Thus, dichotomy restricted to $k$-persistent reals is provable in a constructive axiom system.

Theorem $8\left(\widehat{\mathrm{E}-\mathrm{HA}}_{\uparrow}^{\omega}\right)$ If $\alpha$ is $k$-persistent, then either $\alpha \leq 0$ or $\alpha \geq 0$.

Proof If $\alpha$ is $k$-persistent, consider $\alpha(k)$. Since comparisons of $\alpha(k)$ with 0 are quantifier free formulas, $\widehat{\mathrm{E}-\mathrm{HA}}_{\uparrow}^{\omega}$ proves that $\alpha(k) \leq 0$ or that $\alpha(k) \geq 0$. Consider the case when $\alpha(k) \leq 0$. Suppose that there exists a $j$ such that $\alpha(j)-0>2^{-j+1}$. Due to the rate of convergence of $\alpha$, for all $t>j, \alpha(t)>0$, contradicting the $k$-persistence of $\alpha$. Thus $\neg(0<\alpha)$, which is $\alpha \leq 0$. Similarly, in the case that $\alpha(k) \geq 0$, we have $\alpha \geq 0$.

Restricting item 2 of Theorem 1 to $k$-persistent reals yields a sequential statement that is provable in $\mathrm{RCA}_{0}$.

Theorem $9\left(\mathrm{RCA}_{0}\right)$ Fix $k \in \mathbb{N}$. If $\left\langle\alpha_{i}\right\rangle_{i \in \mathbb{N}}$ is a sequence of $k$-persistent reals, then there is a set $I \subset \mathbb{N}$ such that for all $i, i \in I$ implies $\alpha_{i} \leq 0$ and $i \notin I$ implies $\alpha_{i} \geq 0$.

Proof If $\left\langle\alpha_{i}\right\rangle_{i \in \mathbb{N}}$ is a sequence of $k$-persistent reals, then the set $I \subset \mathbb{N}$ defined by $I=\left\{i \in \mathbb{N} \mid \alpha_{i}(k) \leq 0\right\}$ exists by $\Delta_{1}^{0}$ comprehension and satisfies the theorem. One could also prove this result by applying Theorem 3.6 of Hirst and Mummert [5] to Theorem 8 .

Next, we present analogous restrictions for trichotomy. 
Definition 10 Let $\alpha$ be a real number. We say that $\alpha$ is contractive if for every $s$ and $t>s$, either $\alpha(s) \leq \alpha(t) \leq \alpha(s+1)$ or $\alpha(s+1) \leq \alpha(t) \leq \alpha(s)$.

Informally, each successive pair of rationals in a contractive real provide upper and lower bounds for the value of the real. Reals that are $k$-persistent and contractive have the added feature that if $\alpha(k)$ and $\alpha(k+1)$ are not both positive or both negative, then $\alpha=0$. Consequently, trichotomy for $k$-persistent contractive reals can be proved constructively.

Theorem $11\left(\widehat{\mathrm{E}-\mathrm{HA}}_{\uparrow}^{\omega}\right)$ If $\alpha \geq 0$ is $k$-persistent and contractive, then either $\alpha=0$ or $\alpha>0$.

Proof Suppose $\alpha \geq 0$ is $k$-persistent and contractive. Note that formulas involving comparisons of specific rational numbers are quantifier free. Thus $\widehat{\mathrm{E}-\mathrm{HA}}_{\uparrow}^{\omega}$ proves that either $(\alpha(k) \leq 0 \vee \alpha(k+1) \leq 0)$ or $(\alpha(k)>0 \wedge \alpha(k+1)>0)$. In the first case, since $\alpha$ is $k$-persistent, we know $\alpha=0$. In the second case, $\alpha>0$ because $\alpha$ is contractive.

Working in $\mathrm{RCA}_{0}$, we can prove item 3 of Theorem 4 for sequences of $k$-persistent contractive reals, as follows.

Theorem $12\left(\mathrm{RCA}_{0}\right)$ If $\left\langle\alpha_{i}\right\rangle_{i \in \mathbb{N}}$ is a sequence of non-negative $k$-persistent contractive reals, then there is a set $I \subset \mathbb{N}$ such that for all $i, i \in I$ implies $\alpha_{i}=0$ and $i \notin I$ implies $\alpha_{i}>0$.

Proof Working in $\mathrm{RCA}_{0}$, suppose $\left\langle\alpha_{i}\right\rangle_{i \in \mathbb{N}}$ is a sequence of non-negative $k$-persistent contractive reals. As above, if $\alpha_{i}(k) \leq 0$ or $\alpha_{i}(k+1) \leq 0$, then $\alpha_{i}=0$. Also, if $\alpha_{i}(k)>0$ and $\alpha_{i}(k+1)>0$, then $\alpha_{i}>0$. Consequently, the set $I$ defined by $I=\left\{i \in \mathbb{N} \mid \alpha_{i}(k) \leq 0 \vee \alpha_{i}(k+1) \leq 0\right\}$ has the desired property and exists by $\Delta_{1}^{0}$ comprehension. (The defining formula is actually quantifier free.) This result could also be derived from Theorem 11 by applying Theorem 3.6 of Hirst and Mummert [5].

In formulating the definitions for $k$-persistent and contractive reals, the authors initially searched for computable restrictions of the sequential statements, and then verified the constructive proofs of the principles for individual reals. Thus, working in the reverse mathematics framework can help indicate potential constructive results. Of course, actual constructive results immediately yield proofs of computable restrictions of sequential statements, at least for formulas in $\Gamma_{1}$. 


\section{Variations on persistence}

We say that a real is eventually persistent if it is $k$-persistent for some $k$. RCA 0 proves that every real is eventually persistent, so Theorem 1 holds with sequences of eventually persistent reals substituted in item 2. Examination of the proof of the related computable restriction in Theorem 9 shows that it does not rely on the assumption that every real in the sequence is $k$-persistent for the same $k$. We only need to know some value for $k$ for each real. This information can be encoded in an auxiliary function. We say that $h: \mathbb{N} \rightarrow \mathbb{N}$ is a modulus of persistence for the sequence of reals $\left\langle\alpha_{i}\right\rangle_{i \in \mathbb{N}}$ if for each $i$, $\alpha_{i}$ is $h(i)$-persistent.

Theorem $13\left(\mathrm{RCA}_{0}\right)$ If $h$ is a modulus of persistence for the sequence of reals $\left\langle\alpha_{i}\right\rangle_{i \in \mathbb{N}}$, then there is a set $I \subset \mathbb{N}$ such that for all $i, i \in I$ implies $\alpha_{i} \leq 0$ and $i \notin I$ implies $\alpha_{i} \geq 0$.

Proof Use the proof of Theorem 9, replacing $k$ by $h(i)$.

In the development of real analysis in reverse mathematics [8], many results that require $W_{K} L_{0}$ can be proved in $R_{C A}$ for those special cases where functions have a modulus of uniform continuity. In that setting, the existence of the modulus is equivalent to $W K L_{0}$. By contrast, the existence of moduli of persistence is unexpectedly strong.

Theorem $14\left(\mathrm{RCA}_{0}\right)$ The following are equivalent:

(1) $\mathrm{ACA}_{0}$

(2) Every sequence of reals has a modulus of persistence.

Proof Given a sequence of reals, a modulus of persistence can be easily be defined by an arithmetical formula using the sequence as a parameter. Thus, ACA $A_{0}$ proves item 2.

To prove the reversal, we use item 2 to define the range of an injection. Suppose $f: \mathbb{N} \rightarrow \mathbb{N}$ is one-to-one. Using the bracket notation from the proof of Theorem 4, define a sequence of reals $\left\langle\alpha_{i}\right\rangle_{i \in \mathbb{N}}$ by

$$
\alpha_{i}(n)= \begin{cases}2^{-f^{-1}(i)} & \text { if } i \in f[n] \\ 0 & \text { if } i \notin f[n] .\end{cases}
$$

Apply item 2 to find a modulus of persistence $h$ for $\left\langle\alpha_{i}\right\rangle_{i \in \mathbb{N}}$. By $\Delta_{1}^{0}$ comprehension, the set $X=\{i \mid(\exists k \leq h(i))(f(k)=i)\}$ exists. Clearly, $X$ is a subset of the range of $f$. Conversely, if for some $k$ we have $f(k)=i$, then for any value $j$ less than $k, \alpha_{i}$ is not lower $j$-persistent. Thus, $k \leq h(i)$, and $i$ will be included in $X$. 
Even though $\mathrm{WKL}_{0}$ is too weak to prove the existence of a modulus of persistence for a sequence of reals, it does suffice to prove that every sequence of reals is term-wise equal to a sequence that has a modulus of persistence.

Theorem $15\left(R C A_{0}\right)$ The following are equivalent:

(1) $W K L_{0}$.

(2) If $\left\langle\alpha_{i}\right\rangle_{i \in \mathbb{N}}$ is a sequence of reals, then there is a sequence $\left\langle\beta_{i}\right\rangle_{i \in \mathbb{N}}$ of 0 -persistent reals such that for all $i \in \mathbb{N}, \alpha_{i}=\beta_{i}$.

(3) If $\left\langle\alpha_{i}\right\rangle_{i \in \mathbb{N}}$ is a sequence of reals, then there is a sequence $\left\langle\beta_{i}\right\rangle_{i \in \mathbb{N}}$ of reals with a modulus of persistence such that for all $i \in \mathbb{N}, \alpha_{i}=\beta_{i}$.

Proof To show that $\mathrm{WKL}_{0}$ proves item 2, suppose $\left\langle\alpha_{i}\right\rangle_{i \in \mathbb{N}}$ is a sequence of reals. Using $\mathrm{WKL}_{0}$, apply Theorem 1 to find a set $I$ such that for all $i, i \in I$ implies $\alpha_{i} \leq 0$ and $i \notin I$ implies $\alpha_{i} \geq 0$. Define $\beta_{i}$ as follows. If $i \in I$ and $j \in \mathbb{N}$, let $\beta_{i}(j)=\alpha_{i}(j+1)$ if $\alpha_{i}(j+1)<0$ and let $\beta_{i}(j)=-2^{-j-1}$ otherwise. If $i \notin I$ and $j \in \mathbb{N}$, let $\beta_{i}(j)=\alpha_{i}(j+1)$ if $\alpha_{i}(j+1)>0$ and let $\beta_{i}(j)=2^{-j-1}$ otherwise. Straightforward arguments verify that for each $i, \alpha_{i}=\beta_{i}$ and that $\beta_{i}$ is 0 -persistent.

Since the constant 0 function is a modulus of persistence of the sequence $\left\langle\beta_{i}\right\rangle_{i \in \mathbb{N}}$ of item 2 , clearly item 2 implies item 3 . We complete the proof by deducing $W K L_{0}$ from item 3, finding a separating set for the ranges of injections with disjoint ranges. Suppose $f$ and $g$ are one-to-one functions with disjoint ranges. Construct $\left\langle\alpha_{i}\right\rangle_{i \in \mathbb{N}}$ as in the proof of Theorem 1. Apply item 3 to find a term-wise equal sequence of reals with a modulus of persistence, and then apply Theorem 13 to find the set $I$. As in the proof of Theorem $1, I$ contains the range of $g$ and excludes the range of $f$.

Our choice of the concept of persistence is by no means unique. For example, one can prove item 2 of Theorem 1 in $\mathrm{RCA}_{0}$ provided that the sequence consists only of non-zero reals. Alternatively, one could consider the following definition. A real $\alpha$ is sticky if

- $\forall i((\alpha(i) \geq 0 \wedge \alpha(i+1) \geq 0) \rightarrow \alpha(i+2) \geq 0)$,

- $\forall i((\alpha(i) \leq 0 \wedge \alpha(i+1) \leq 0) \rightarrow \alpha(i+2) \leq 0)$, and

- $\exists i((\alpha(i) \geq 0 \wedge \alpha(i+1) \geq 0) \vee(\alpha(i) \leq 0 \wedge \alpha(i+1) \leq 0))$.

Intuitively, if $\alpha$ is sticky and we plot pairs $(i, \alpha(i))$ for all $i$, the graph may alternate above and below the axis for a while, but eventually it will stick above the axis or below the axis. The preceding results all hold with 0 -persistence or $k$-persistence replaced by stickiness; we leave these proofs as exercises for aficionados of sticky things. 


\section{Relatively persistent reals}

Rather than comparing a real to 0 as in the definition of $k$-persistent, we can compare a pair of reals. If $\alpha$ and $\beta$ are reals, we say that $\alpha$ and $\beta$ are relatively $k$-persistent if $\alpha-\beta$ is $k$-persistent. Here $\alpha-\beta$ is shorthand for $\alpha+(-\beta)$, and addition and unary negation on reals are defined as in Definition II.4.4 of Simpson [8]. Note that RCA proves that $\alpha-\beta$ is $k$-persistent if and only if $\beta-\alpha$ is $k$-persistent, so the definition does not depend on the order of the reals. Consider the following result on minima.

Theorem $16\left(\mathrm{RCA}_{0}\right)$ The following are equivalent:

(1) $\mathrm{WKL}_{0}$.

(2) If $\left\langle\left\langle\alpha_{i}^{j}\right\rangle_{i \leq n_{j}}\right\rangle_{j \in \mathbb{N}}$ is a sequence of finite sequences of reals, then there is a function $h: \mathbb{N} \rightarrow \mathbb{N}$ such that for every $j \in \mathbb{N}, \alpha_{h(j)}^{j}$ is a minimum for $\left\langle\alpha_{i}^{j}\right\rangle_{i \leq n_{j}}$. That is, $\alpha_{h(j)}^{j} \leq \alpha_{i}^{j}$ for every $i \leq n_{j}$.

(3) If $\left\langle\left(\alpha_{0}^{j}, \alpha_{1}^{j}\right)\right\rangle_{j \in \mathbb{N}}$ is a sequence of ordered pairs of reals, then there is a function $h: \mathbb{N} \rightarrow 2$ such that for every $j \in \mathbb{N}, \alpha_{h(j)}^{j}$ is a minimum of $\left(\alpha_{0}^{j}, \alpha_{1}^{j}\right)$.

(4) If $\left\langle\alpha_{i}\right\rangle_{i \in \mathbb{N}}$ is a sequence of reals then there is a function $h: \mathbb{N} \rightarrow \mathbb{N}$ such that for every $i \in \mathbb{N}, \alpha_{h(i)}$ is a minimum of $\left\langle\alpha_{j}\right\rangle_{j \leq i}$.

Proof Working in $\mathrm{RCA}_{0}$, to prove that $\mathrm{WKL}_{0}$ implies item 2 , construct a tree $T \subset \mathbb{N}<\mathbb{N}$ as follows. Place the sequence $\sigma$ of length $l$ in $T$ if and only if for each $j<l$ there is no witness below $l$ that $\alpha_{\sigma(j)}^{j}$ is not the minimum of $\left\langle\alpha_{i}^{j}\right\rangle_{i \leq n_{j}}$. More precisely, $\sigma$ is in $T$ if and only if for every $j<l$ there is no $i \leq l$ and $m \leq n_{j}$ such that $\alpha_{\sigma(j)}^{j}(i)-2^{-i+1}>\alpha_{m}^{j}(i) . \mathrm{RCA}_{0}$ can prove that $T$ is infinite and that the labels used in level $j$ of the tree are bounded by $n_{j}$. Using $\mathrm{WKL}_{0}$, apply Lemma IV.1.4 of Simpson [8] to find an infinite path through $T$. The sequence of nodes in the path gives a sequence of values for a function $h$ that satisfies item 2 .

Clearly, item 3 is a special case of 2 . To see that item 3 implies $W K L_{0}$, we use Theorem 1. let $\left\langle\alpha_{j}\right\rangle_{j \in \mathbb{N}}$ be a sequence of reals and consider the sequence of ordered pairs $\left\langle\left(\beta_{0}^{j}, \beta_{1}^{j}\right)\right\rangle_{j \in \mathbb{N}}$ where for all $j, \beta_{0}^{j}=0$ and $\beta_{1}^{j}=\alpha_{j}$. Apply item 3 and use $\Delta_{1}^{0}$-comprehension to prove the existence of $I=\{i \in \mathbb{N} \mid h(i)=1\}$. Then $I$ satisfies item 2 of Theorem 1, which is equivalent to $\mathrm{WKL}_{0}$.

Item 4 is also a special case of item 2, with the finite sequences taken as initial segments of a single infinite sequence. The proof that item 4 implies $W_{K} L_{0}$ is part of Theorem 2 of Hirst [4]. 
Applying the concept of relatively $k$-persistent, we can formulate a computable restriction of item 2 of the preceding theorem.

Theorem $17\left(\mathrm{RCA}_{0}\right)$ If $\left\langle\left\langle\alpha_{i}^{j}\right\rangle_{i \leq n_{j}}\right\rangle_{j \in \mathbb{N}}$ is a sequence of finite sequences of reals such that each finite sequence is pairwise relatively $k$-persistent, then there is a function $h: \mathbb{N} \rightarrow \mathbb{N}$ such that for every $j \in \mathbb{N}, \alpha_{h(j)}^{j}$ is a minimum of $\left\langle\alpha_{i}^{j}\right\rangle_{i \leq n_{j}}$.

Proof Working in $\mathrm{RCA}_{0}$, use the following process to compute $h(j)$ from $\left\langle\alpha_{i}^{j}\right\rangle_{i \leq n_{j}}$. Linearly order the elements of $\left\langle\alpha_{i}^{j}\right\rangle_{i \leq n_{j}}$ by starting with $\alpha_{0}^{j}$. If $\alpha_{0}^{j}, \ldots, \alpha_{i}^{j}$ have been linearly ordered, place $\alpha_{i+1}^{j}$ just to the left of the leftmost $\alpha_{i^{\prime}}^{j}$ such that $\alpha_{i^{\prime}}^{j}(k)-\alpha_{i+1}^{j}(k) \geq$ 0 . (By relative $k$-persistence, $\alpha_{i^{\prime}}^{j} \geq \alpha_{i+1}^{j}$.) If no such $i^{\prime}$ exists, place $\alpha_{i+1}^{j}$ on the extreme right. When all $n_{j}$ elements have been linearly ordered, let $h(j)$ be the index of the leftmost real.

The process described in the preceding proof can be executed in a weak constructive setting, proving the following result.

Theorem $18\left(\widehat{\mathrm{E}-\mathrm{HA}}^{\omega}+\mathrm{QF}-\mathrm{AC} \mathrm{C}^{0,0}\right)$ Every finite sequence of pairwise relatively $k$-persistent reals has a minimum.

The finite linear orderings in the proof of Theorem 17 can be thought of as finite approximations of embeddings of sequences of reals in $\mathbb{Q}$. Consequently, it is natural to rephrase Theorem 16 as follows.

Theorem $19\left(\mathrm{RCA}_{0}\right)$ The following are equivalent:

(1) $\mathrm{WKL}_{0}$.

(2) If $\left\langle\left\langle\alpha_{i}^{j}\right\rangle_{i \leq n_{j}}\right\rangle_{j \in \mathbb{N}}$ is a sequence of finite sequences of reals, then there is a sequence of embeddings $e_{j}: n_{j}+1 \rightarrow \mathbb{N}$ such that for all $i$ and $i^{\prime}$ less than or equal to $n_{j}$, $e_{j}(i) \leq e_{j}\left(i^{\prime}\right)$ implies $\alpha_{i}^{j} \leq \alpha_{i^{\prime}}^{j}$.

(3) For every sequence of reals $\left\langle\alpha_{i}\right\rangle_{i \in \mathbb{N}}$ there is an embedding $e: \mathbb{N} \rightarrow \mathbb{Q}$ such that for all $i$ and $j, e(i) \leq e(j)$ implies $\alpha_{i} \leq \alpha_{j}$.

Proof To prove that $\mathrm{WKL}_{0}$ proves item 2, use $\mathrm{WKL}_{0}$ and apply Theorem 15 to find 0 -persistent forms of all the pairwise differences of the reals from each finite sequence, acquiring essentially the same information as if each pair was relatively 0 -persistent. Use the construction of Theorem 17 to find the linear orderings. 
To prove that item 2 implies item 3, apply item 2 to the sequence of initial segments of $\left\langle\alpha_{i}\right\rangle_{i \in \mathbb{N}}$ and find linear orderings for each initial segment. Gradually construct $e$ as follows. Suppose $e$ is defined on $\alpha_{0}, \ldots, \alpha_{j}$. To simplify notation, renumber the indices so that the embedded order matches the order of the indices. If the finite ordering provided by item 2 on $\alpha_{0}, \ldots, \alpha_{j}, \alpha_{j+1}$ indicates that $\alpha_{j+1} \leq \alpha_{0}$, let $e(j+1)=e(0)-1$. If it indicates that $\alpha_{j} \leq \alpha_{j+1}$, let $e(j+1)=e(j)+1$. Otherwise, find the least $i$ such that $\alpha_{i} \leq \alpha_{j+1} \leq \alpha_{i+1}$ or $\alpha_{i+1} \leq \alpha_{j+1} \leq \alpha_{i}$, and let $e(j+1)=(e(i)+e(i+1)) / 2$.

To show that item 3 implies $W K L_{0}$, deduce item 2 of Theorem 16 by using the embedding from item 3 to select minima of initial segments of a sequence of reals.

Following the pattern of Theorem 17 and Theorem 18, we could use relative 0 persistence to formulate computable and constructive analogs of item 2 of Theorem 19. If we require that the embeddings of Theorem 19 preserve equality, the corresponding result acts more like trichotomy and requires $A C A_{0}$.

Theorem $20\left(\mathrm{RCA}_{0}\right)$ The following are equivalent:

(1) $\mathrm{ACA}_{0}$

(2) If $\left\langle\left\langle\alpha_{i}^{j}\right\rangle_{i \leq n_{j}}\right\rangle_{j \in \mathbb{N}}$ is a sequence of finite sequences of reals, then there is a sequence of embeddings $e_{j}: n_{j}+1 \rightarrow \mathbb{N}$ such that for all $i$ and $i^{\prime}$ less than or equal to $n_{j}$, $e_{j}(i) \leq e_{j}\left(i^{\prime}\right)$ if and only if $\alpha_{i}^{j} \leq \alpha_{i^{\prime}}^{j}$.

(3) For every sequence of reals $\left\langle\alpha_{i}\right\rangle_{i \in \mathbb{N}}$ there is an embedding $e: \mathbb{N} \rightarrow \mathbb{Q}$ such that for all $i$ and $j, e(i) \leq e(j)$ if and only if $\alpha_{i} \leq \alpha_{j}$.

Sketch of proof To show that $\mathrm{ACA}_{0}$ proves item 2, it is easy to show that the desired sequence of embeddings is arithmetically definable. The proof that item 2 implies item 3 can be adapted from the similar proof for Theorem 19. To complete the proof, it suffices to use item 3 to derive item 3 of Theorem 4 , since that statement implies $\mathrm{ACA}_{0}$. Given a sequence of reals $\left\langle\alpha_{i}\right\rangle_{i \in \mathbb{N}}$, define the sequence $\left\langle\beta_{i}\right\rangle_{i \in \mathbb{N}}$ by $\beta_{0}=0$ and $\beta_{i+1}=\alpha_{i}$ for all $i$. Applying item 3 to $\left\langle\beta_{i}\right\rangle_{i \in \mathbb{N}}$, we know that $\alpha_{i}=0$ if and only if $e(i+1)=e(0)$.

As a final exercise, the reader could define the notion of relatively $k$-persistent contractive pairs of reals and formulate and prove computable and constructive restrictions of item 2 of Theorem 20. 
F G Dorais, JL Hirst and P Shafer

\section{Acknowledgements}

Jeffry Hirst was supported by grant (ID\#20800) from the John Templeton Foundation. The opinions expressed in this publication are those of the authors and do not necessarily reflect the views of the John Templeton Foundation.

\section{References}

[1] V Brattka, G Gherardi, Effective choice and boundedness principles in computable analysis, Bull. Symbolic Logic 17 (2011), 73-117; doi:10.2178/bsl/1294186663.

[2] D Bridges, F Richman, Varieties of constructive mathematics, London Mathematical Society Lecture Note Series Vol 97, Cambridge University Press, Cambridge, 1987; doi: 10.1017/CBO9780511565663.

[3] D S Bridges, L S Vîţă, Techniques of constructive analysis, Universitext, Springer, New York, 2006; doi:10.1007/978-0-387-38147-3.

[4] J L Hirst, Minima of initial segments of infinite sequences of reals, Math. Log. Quarterly 50 (2004), 47-50; doi:10.1002/malq.200310075.

[5] J L Hirst, C Mummert, Reverse mathematics and uniformity in proofs without excluded middle, Notre Dame J. Form. Log. 52 (2011), 149-162; doi:10.1215/00294527-1306163.

[6] H Ishihara, An omniscience principle, the König lemma and the Hahn-Banach theorem, Z. Math. Logik Grundlag. Math. 36 (1990), 237-240; doi:10.1002/malq.19900360307.

[7] U Kohlenbach, Applied proof theory: proof interpretations and their use in mathematics, Springer Monographs in Mathematics, Springer-Verlag, Berlin 2008; doi:10.1007/9783-540-77533-1.

[8] SG Simpson, Subsystems of second order arithmetic, second edition, Perspectives in Logic, Cambridge University Press, Cambridge, 2009; doi:10.1017/CBO9780511581007.

Department of Mathematics, Dartmouth College, 6188 Kemeny Hall Hanover, NH 03755

Department of Mathematical Sciences, Appalachian State University, Boone, NC 28608

francois.g.dorais@dartmouth.edu, jlh@math.appstate.edu, paul.shafer@gmail.com

http://www.appstate.edu/ doraisfg/, http://mathsci2.appstate.edu/ jlh/, http://www.appstate.edu/ shaferpe/

Received: 28 August 2011 Revised: 22 February 2012 